\title{
Utility of invasive urodynamics before surgery for stress urinary incontinence
}

\author{
Gunnar Lose • Niels Klarskov
}

Received: 12 June 2013 / Accepted: 15 June 2013 / Published online: 4 July 2013

(C) The International Urogynecological Association 2013

The utility of invasive urodynamic testing in the preoperative evaluation of women with stress urinary incontinence (SUI) has been challenged by two recent randomized controlled trials (RCTs) $[1,2]$ that could not document any gain on outcome of surgery. However, both studies have common methodological issues that make it difficult to draw a general conclusion.

Urodynamics (UDS) is defined by the International Continence Society (ICS) as the study of the function and dysfunction of the urinary tract by any appropriate method [3]. Accordingly, UDS is the only way to understand why people are continent or incontinent. Treatment that is not carried out blindfolded but based on knowledge requires (noninvasive and/or invasive) UDS [4].

It is well documented that invasive urodynamic testing (in terms of cystometry and pressure-flow study) in women with predominant symptomatic SUI may show either detrusor overactivity (DO) solely or genuine SUI in combination with other urodynamic findings, such as DO and/or voiding difficulties (in terms of obstruction or hypoactive detrusor function). In addition, urethral profilometry or leak-point pressure allows identification of individuals with low urethral pressure [i.e., intrinsic sphincter deficiency (ISD)]. The surgeon who wishes to acquire this knowledge needs to use invasive UDS.

Invasive UDS has principally two goals: to confirm clinical and noninvasive UDS impression; to identify parameters that may alter diagnosis or treatment. However, prerequisites for taking advantage of invasive UDS imply:

1. Performing the most appropriate UDS, and secure highquality performance and expertise in interpretation.

2. Clear definition of urodynamic entities (i.e., clear cutoff values for urodynamic parameters), which make subcategorizing possible; e.g., obstruction or hyperactive detrusor function.

G. Lose $(\bowtie) \cdot$ N. Klarskov

Department of Obstetrics \& Gynecology, Pavillon B, Herlev Hospital, Herlev Ringvej, 2730 Herlev, Denmark

e-mail: Gunnar.Lose@regionh.dk
3. Evidence/knowledge-based stratified therapeutic strategy based on urodynamic findings.

The authors of the two papers deserve much credit for trying to answer the research question regarding the utility of invasive UDS before surgery for female SUI. However, both studies have common conceptual flaws:

\section{Choice of UDS, quality, and expertise}

The choice of UDS was different in the two studies.

None of the study centers seem to be UDS-certified by the ICS (or another organization), although there are references to the ICS and the principles of good urodynamic practice [5]. The Dutch study [2] includes one academic and nine nonacademic hospitals. Altogether, 31 patients had invasive UDS (about three per center). It is remarkable that only $52 \%$ of patients $(16 / 31)$ had urodynamically demonstrable SUI. Furthermore, some values given in Table 2 of that study are obviously outliers (i.e., artefacts): e.g., maximal urethral closing pressure $212 \mathrm{mmHg}$.

In the Value of Urodynamic Evaluation study (ValUE) [1], 11 centers participated. Urodynamic SUI was demonstrated in $97 \%$ of patients.

In both studies, UDS quality and the level of urodynamic expertise are unclear.

2. No clear definition of urodynamic entities

There was no clear definition of DO, overactive bladder with/without incontinence, hypoactive detrusor function, obstruction, voiding dysfunction, ISD, weak flow, postvoid residual (PVR), and small cystometric capacity. These terms require clear cutoff values. Interpretation and urodynamic categorization seem to be based on individual, subjective judgement by the study physician at each center.

\section{Treatment strategy}

Neither study outlines a clear treatment strategy based on urodynamic findings (i.e., urodynamic answer). Surgery was cancelled in a few cases; in other cases, conservative treatment was tried, and in some patients, the 
surgical approach was modified [1]. Decision making, however, seems to be subjective and individual.

UDS includes a broad spectrum of noninvasive/invasive tests. The fundamental concept of UDS is to formulate urodynamic question/s, choose the relevant tests, interpret the urodynamic answer/s, and initiate treatment accordingly. The two recent RCTs show that the involved centers (altogether) did not gain anything from UDS in relation to SUI treatment outcome based on (1) their choice of UDS (noninvasive/invasive), and (2) a subjective and nonuniform interpretation of tests and treatment strategy at each center. However, a design such as this does not allow any global conclusion, because it has inborn components of bias. Thus, the lack of gain may be owing to: (a) the fact that the doctors did not actually utilize UDS because of poor quality, low expertise in interpretation, or aversion; (b) wrong choice of UDS (or cutoff levels, if any), (c) lack of clear evidence/knowledgebased therapeutic algorithms; (d) choice of outcome measures. Consequently, if one or more of these critical factors were changed, another study may provide another result. Furthermore, as the doctors essentially did the same intervention (surgical treatment) in both arms, no effect of UDS is to be expected. Therefore, neither study allows for the conclusion: "preoperative urodynamic testing does not improve the outcome of surgery for stress urinary incontinence."

It is clear from the reported information that there was a tendency to perform surgery independent of urodynamic findings ( $93 \%$ in the ValUE study versus $94 \%$ in the Dutch study). The Dutch study actually reported aversion against UDS (“...some specialists had already stopped performing urodynamics in this patient category..."), and the study was terminated because of inclusion difficulties [2].

There is a huge body of evidence indicating that urethral slings cannot be performed successfully in all patients with predominant SUI, irrespective of UDS findings such as DO, voiding difficulties, and other urodynamic diagnoses. It is well documented that women with preoperative DO are more likely to experience treatment failure following midurethral slings (MUS), and much evidence indicates that urodynamic quantification of $\mathrm{DO}$ would be a stronger predictor of surgery outcome than the present qualitative definition of DO by ICS/International Urogynecological Association (IUGA) [6]. It is also well documented that MUS is an obstructive procedure [7] and that postsurgical voiding dysfunction occurs in $5-15 \%$ of patients, which constitutes a substantial problem [8]. Intuitively, it seems logical to take a diagnosis of (preoperative) voiding dysfunction (hypoactive detrusor and/or obstruction) into consideration when surgery is planned. At the present moment, we do not know which parameters are the most sensitive by which to characterize bladder emptying phase (maximum flow rate, detrusor pressure, flow time, postvoid residual, straining, or any combination). The Dutch study showed that voiding dysfunction after surgery appeared less often in the group of women who underwent invasive UDS: $3 / 33$ (9.6 \%) versus
$7 / 28(25 \%)$ in the group without UDS. However, the study was severely underpowered, and the finding did not reach statistical significance. Follow-up by the ValUE study showed that only $62.1 \%$ of patients who had voiding dysfunction before surgery obtained successful outcome after surgery versus $78.3 \%$ of women without voiding dysfunction before surgery (0.064) [9].

Studies indicate that decreased flow rate [10], PVR [11], and straining [12] may play a predictive role. Urethral pressure profilometry or leak-point pressure may show a low urethral pressure (low-pressure urethra), which may be useful in counselling the patient about her prognosis and helpful for the surgeon in choosing the right procedure or sling tension [13].

The development of UDS, which makes good sense in understanding the underlying function/dysfunction, has made scant progress over the last 2 decades, possibly because of the successful introduction of the MUS. However, there is a need to develop UDS and reappraisal of its utility in predicting surgical outcome. New studies should be based on documented UDS quality, consensus regarding defined urodynamic entities (i.e., cutoff levels and standards), and clearly documented treatment strategies linked to UDS findings. This is fundamental in order to test UDS utility and to communicate results in a meaningful way.

\section{References}

1. Nager CW, Brubaker L et al (2012) A randomized trial of urodynamic testing before stress-incontinence surgery. N Engl J Med 366(21):1987-1997

2. Van Leijsen SAL, Kluivers KB et al (2012) Can preoperative urodynamic investigation be omitted in women with stress urinary incontinence? A non-inferiority randomized controlled trial. Neurourol Urodyn 31(7):1118-1123

3. Abrams P, Blaivas JG, Stanton S et al (1988) Standardisation of terminology of lower urinary tract function. Neurourol Urodyn $7: 403-427$

4. Griffiths D, Kondo A, Bauer S, Diamant N, Liao L, Lose G et al (2005) Dynamic testing. In: Abrams N, Cardozo L, Khoury S, Wein A (eds) Incontinence. 3rd international consultations on incontinence. Health Publications Ltd, Plymouth, pp 587-673

5. Schäfer W, Abrams P et al (2002) Good urodynamic practices: uroflowmetry, filling cystometry, and pressure-flow studies. Neurourol Urodyn 21(3):261-274

6. Lose G, Brostrøm S (2010) Current evidence and practice in female mixed incontinence. Eur Obstet Gynaecol 5:61-64

7. Wang AC, Chen MC (2003) The correlation between preoperative voiding mechanism and surgical outcome of the tension-free vaginal tape procedure, with reference to quality of life. BJU Int 91(6):502-506

8. Rodrigues P, Hering F, Dias EC (2013) Female obstruction after incontinence surgery may present different urodynamic patterns. Int Urogynecol J 24(2):331-336

9. Sirls LT, Richter HE, Litman HJ, Kenton K et al (2013) The effect of urodynamic testing on clinical diagnosis, treatment plan and outcomes in women undergoing stress urinary incontinence surgery. J Urol 189:204-209

10. Rosier PF, Gajewski JB et al (2010) Executive summary: the international consultation on incontinence 2008 - Committee on:"dynamic 
testing"; for urinary incontinence and for fecal incontinence. Part 1: innovations in urodynamic techniques and urodynamic testing for signs and symptoms of urinary incontinence in female patients. Neurourol Urodyn 29:140-145

11. De Almeida SH, Gregorio E et al (2004) Variables predictive of voiding disfunction following aponeurotic sling surgery: multimariate analysis. Int Braz J Urol 30:302-306
12. Pham KN, Topp N et al (2010) Preoperative Valsalva voiding increases the risk of urinary retention after midurethral sling lacement. Int Urogynecol J 21:1243-1246

13. Schierlitz L, Dwyer PL, Rosamilia A et al (2012) Three-year followup of tension-free vaginal tape compared with transobturator tape in women with stress urinary incontinence and intrinsic sphincter deficiency: a randomized controlled trial. Obstet Gynecol 119:321-327 